\title{
Performance Comparison of Routing Protocols of MANET in Real World Scenario using NS3
}

\author{
Supriya Singla \\ Computer Science and Engineering department \\ Thapar University \\ Patiala, India
}

\author{
Sushma Jain \\ Computer Science and Engineering department \\ Thapar University \\ Patiala, India
}

\begin{abstract}
Mobile ad hoc network (MANET) is a collection of several wireless devices or mobile users that can communicate among themselves over wireless links in a peer to peer basis and thereby creating a dynamic, arbitrary graph. But some adverse characteristics of MANET like dynamic topology, limited bandwidth, link failure and energy constraints, imposes new demands on routing protocol. This paper aims to study the performance evaluation and comparison of three prominent routing protocols: Destination Sequence Distance Vector (DSDV), Ad-hoc On demand Distance Vector (AODV) and Optimized Link State Routing (OLSR), in a real life scenario. In a given scenario, students investigate the historical site in which number of packets being sends and number of nodes in the network affects the communication reliability. Extensive simulations are made to evaluate the performance of these protocols using various performance differential metrics like packet delivery ratio, total energy consumption and throughput using NS3. In the end it is seen that in most simulation results, proactive routing protocols (DSDV, OLSR) performed significantly better than reactive routing protocols.
\end{abstract}

\section{Keywords}

Ad hoc; Routing Protocols; AODV; DSDV; OLSR; Scenario; Performance comparison; NS3.

\section{INTRODUCTION}

Evolution of wireless and mobile communication, enables users use their cellular phones to browse the Internet anywhere but these networks require centralized administration with fixed network infrastructure, that also includes set-up and maintenance cost. But, easy availability of mobile nodes enabled with short range wireless interface and computation capability gave the concept of MANET. A Mobile ad hoc Network (MANET) [11] is a network of portable autonomous nodes equipped with wireless interface, communicates over wireless links without centralized administration. These networks can self-configure and maintain the network topology dynamically without the infrastructural support.

MANETs have many applications: in tactical networks, emergency services, commercial and civilian environments, home and enterprise networking, education, entertainment, sensor networks, context aware services and coverage extension [12]. Figure 1 shows an ad hoc network with three wireless mobile hosts. Node 1 is not within the range of node 3 's wireless transmitter and vice versa. If node 1 and node 3 want to exchange packets, they must enlist the services of node 2 to forward the packets for them, since node 2 is within the range overlap between node 1 and node 3 .

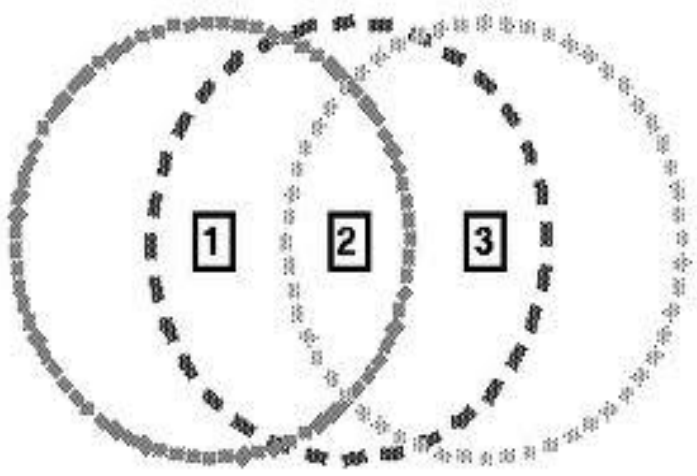

Fig 1: Simple ad-hoc network with three participating nodes

The infrastructureless design, dynamic nature, the bandwidth constrained, variable capacity of links and the network scalability, demands new set of strategies in their routing protocols to provide efficient end-to-end communication. Each protocol uses different metrics to find feasible path to reach the destination. Further each routing protocols performance is different in different scenarios due to their different methodologies of route creation and route maintenance. So it is difficult to determine which protocols may perform best under a number of different network scenarios. Simulations made till now are not based on real life scenarios. This is probably the main reason that MANETs have not been used extensively in day to day applications although they have significant advantages above traditional communication networks [13].

In this paper, in a given scenario, students investigate the historical site in which number of packets being sends and number of nodes in the network affects the communication reliability. Extensive simulations are made using various performance differential metrics like packet delivery ratio, total energy consumption and throughput. For simulations NS3 is being used. In the end it is seen that in most simulation results, proactive routing protocols (DSDV, OLSR) performed significantly better than reactive routing protocols.

The rest of the paper is organized as follows. In section 2 literatures survey is presented. In Section 3 brief overview of routing protocols are pointed out. Section 4, describes real world scenario taken into consideration. Simulation results in a given scenario are shown in section 5. Finally, conclusions are drawn in section 6 . 


\section{LITERATURE SURVEY}

Routing protocols performance depends on network environment like node density, traffic, mobility, pause time, type of traffic etc. In [10] results shown that proactive protocols provide excellent performance in terms of packet delivery ratio and end-to-end delays and reactive protocols shows good performance in terms of routing load. In [4] it was concluded that in less dense networks, STAR achieves better overall performance than AODV and DSR but during highly dense networks STAR performs much better in terms of control overhead while AODV performs better in data delivery. In [8] author concluded that under (constant bit rate) CBR traffic proactive routing protocols are superior than ondemand routing protocols at the cost of higher routing load. AODV is preferred over DSR and DSDV for real time traffic is shown in [1]. In [2] it was concluded that OLSR behavior changes according to the change in mobility model and traffic type (VBR or CBR) used. In [3] author vary different network environments like mobility rate, network load and number of nodes and used Manhattan Grid Mobility Model and concluded that in terms of PDR and Throughput, AODV and DSR perform better than DSDV and in terms of average endto-end delay, DSDV appears to be the best one. In [7] it was concluded that under TCP traffic, DSDV performance is better than AODV but at the cost of higher end-to-end delay in both freeway and random waypoint mobility model.

In [5] author concluded that LAR1 performed much better than expected for high density networks than other ones. DSR resulted least messaging overhead. During high density networks, AODV generated higher volume of control packets even more than the LAR1 but in low density networks, LAR1 generated higher volume of control packets. End-to-end delay was constantly greater in LAR1 than those of the two in sparse networks and was constantly greater in AODV than other two in high density networks, but when the node mobility was increased from 0 to perpetual mobility, LAR1 resulted in the highest increase rate in delay. In [16] author compared the performance of AODV and DSR with respect to average energy consumption and routing energy consumption. Then, an evaluation of how the varying metrics (Number of nodes, Topology size, Packet Rate and Maximum no. of nodes) in different scenarios affect the power consumption in these protocols was discussed. Results concluded that DSR was efficient with most mobility scenarios but at the cost of routing overhead. On the other hand AODV is efficient with some mobility scenarios by eliminating routing overhead of the DSR protocol. It was also shown that DSR resulted better performance with the perspective of energy consumption for low density networks and also for high density networks than AODV. However, AODV was found effective for low loads. They also analyzed that by considering the routing overhead of AODV and reducing the number of control packets, life time of the network can be increased.

\section{ROUTING PROTOCOLS}

Routing protocols are divided into two categories: Reactive and Proactive. Reactive protocols create and maintain routes when they are needed by the source host. On the other hand, Proactive routing protocols maintains updated lists of destinations and their routes by distributing routing tables periodically throughout the network. In this section, AODV, DSDV and OLSR routing protocols are pointed out.

\subsection{Ad hoc on demand distance vector routing protocol}

Ad Hoc on-demand Distance vector [9] (AODV) is a very efficient and effective routing protocol. AODV was motivated by the limited bandwidth of the media used for wireless communication. It uses an on-demand approach for finding routes i.e. routes are created and maintained only when they are needed. It borrows, on demand route discovery and route maintenance from DSR and usage of node sequence numbers from DSDV. The major difference between DSR and AODV stems out from the fact that in DSR, data packet carries the total path to be traversed but in AODV, source node and intermediate nodes store the next hop information and a sequence number which is received from the destination indicating the freshness of the received information (A node updates its path information only if the last DestSeqNum stored at the node is less than the DestSeqNum of the current packet received). Also the information about the active neighbors is received so that when the corresponding route breaks, then the neighbors can be notified.

In an on-demand routing protocol, when a route is not available for the desired destination, source node floods RouteRequest (RREQ) packets to its neighbors with the requested destination sequence number. Multiple routes can be obtained from single RouteRequest. A RouteRequest carries the source identifier (SrcID), the source sequence number (SrcSeqNum), the destination identifier (DestID), the destination sequence number (DestSeqNum) and the broadcast identifier (BcastID) and the time to live field.

When an intermediate node receives a RouteRequest, it either prepares a RouteReply if it has a valid route to the destination or forwards it to its neighbors. BcastID-SrcID pair discards the duplicate copies received at the nodes. Either destination nodes or intermediate nodes having valid routes are allowed to generate RouteReply packets to the source. While forwarding a RouteRequest, every intermediate node enters the previous node address and it's BcastID. So, to maintain the active path at the intermediate nodes, a timer is used to delete the entry in case a RouteReply is not received before the timer expires. On receiving the RouteReply packet, a node stores the information about the previous node from where it receives the packet in order to forward the packet to this next node as the next hop toward the destination. Some of its characteristics are shown in Table 1.

Table 1. AODV Characteristics

\begin{tabular}{|l|l|}
\hline Parameters & Values \\
\hline Routing Architecture & Flat \\
\hline Philosphy & Distributed \\
\hline Type & Reactive \\
\hline Broadcasts & Periodically \\
\hline Route updation & Non-periodic \\
\hline Multicasting & Yes \\
\hline Beacon packets & No \\
\hline Multiple Routes created & No \\
\hline $\begin{array}{l}\text { Utilizes Route Cache/Table } \\
\text { Expiration Timers }\end{array}$ & Yes \\
\hline $\begin{array}{l}\text { Route Maintenance } \\
\text { Methodology }\end{array}$ & Erase route; Notify Source \\
\hline Routing Metric & Fresh and shortest path \\
\hline
\end{tabular}




\subsection{Destination sequenced distance vector}

DSDV [15] is one of the first ad hoc wireless networks protocols. It is a table driven routing protocol meaning that at all times, routes to all destinations is readily available at every node. Every node maintains a table listing all the other nodes it has known directly or through some neighbors. To keep an up-to-date view of the network topology, tables are exchanged between the neighbors at regular intervals. Each table entry contains node's IP address, last known sequence number and the hop count to reach that node. Each node uses 2 mechanisms to send out the DSDV updates. They are periodic updates and trigger updates (these are small updates in-between the periodic updates). Single network data packet unit (NDPU) is used during an incremental update meaning that when a node does not observe significant changes in the local topology, where as multiple NPDUs are used during a full dump meaning that when an incremental update requires more than single NPDU or local topology changes significantly.

Table updates are initiated by a destination with a new sequence number and based on this sequence number; it may forward or reject the table. DSDV uses triggered route updates during topology change. In highly mobile scenarios, a concept of weighted setting time is considered where an update with change in metric is not advertised to neighbors due to great chance of route fluctuations Some of its characteristics are given in Table 2.

\section{Table 2. DSDV Characteristics}

\begin{tabular}{|l|l|}
\hline Parameters & Values \\
\hline Routing Architecture & Flat \\
\hline Loops & No \\
\hline Multicast capability & No \\
\hline No of required tables & 2 \\
\hline Update transmission & Periodically \&as needed \\
\hline Updates transmission & Neighbors \\
\hline Utilizes sequence numbers & Yes \\
\hline Utilizes beacon packets & Yes \\
\hline Overhead & High \\
\hline Routing Metric & Shortest Path \\
\hline
\end{tabular}

\subsection{Optimized Link state routing}

Optimized Link State Protocol (OLSR) [16] is a proactive routing protocol i.e. route is always immediately available when needed. OLSR is an optimized version of classical link state protocol which creates large control packet overhead. OLSR uses Multipoint Relays (MPR) to reduce the information exchange overhead in the network as only the MPRs are allowed to broadcast the packet and MPR set of host is kept small. This is the reason why OLSR is used in high dense network.

In OLSR, Hello and Topology Control (TC) messages are used as control messages where Hello messages are used for performing the task of link sensing, neighbor detection and MPR signaling and TC messages are used for performing the task of topology declaration (advertisement of link states). Another type of message is Multiple Interface Declaration (MID) messages, performs the task of declaring the presence of multiple interfaces on a node and which is broadcasted throughout the entire network by MPRs. There is also a "Host and Network Association" (HNA) message which provides information about the network and netmask addresses. HNA is considered as a generalized version of the TC message with only difference that the HNA message information is removed only after expiration time while TC message inform about the route cancelling. Some of its characteristics are pointed out in Table 3.

Table 3. OLSR Characteristics

\begin{tabular}{|l|l|}
\hline Parameters & Values \\
\hline Routing Architecture & Flat \\
\hline Number of tables & $\begin{array}{l}\text { 3(Routing, neighbor and } \\
\text { topology table) }\end{array}$ \\
\hline Updates Frequency & Periodic \\
\hline Hello messages & Yes \\
\hline Characteristic feature & $\begin{array}{l}\text { Reduces control overhead } \\
\text { using MPR and contention/ } \\
\text { 2-hop neighbor knowledge } \\
\text { required. }\end{array}$ \\
\hline
\end{tabular}

\section{REAL-LIFE SIMULATION SCENARIO}

This scenario describes a trip in historical site performed by a school to provide students a holiday and experiences outside their everyday activities. More specifically, the aim of this scenario is to know about Taj Mahal, a white marble mausoleum, widely recognized as "the jewel of Muslim art in India. In this scenario, a group of nodes which includes students and teachers and 1 tour guide is made. Students were told to analyze the mughal art done by mughals and to know about its history, myths and replicas and to make an assignment on it. Further, it is not allowed to interact with each other i.e. no collaboration is succeeded in any point. They can also take the help from internet to complete their assignment but in Taj Mahal, there is no such established network due to its security and also, 1 tour guide could not be able to guide them properly as lots of external noise may be present. So, we propose to use mobile adhoc network in this group in which, tour guide would be providing them hand held devices through which students can watch videos, images and documents as they reach near any art or something else. So, in this way 1 tour guide can guide so many groups.

MANET is made very easily at low cost and at any time between hand-held cellular based devices having wireless interface and computational power. So MANET is being used in our scenario. In a created scenario we have assumed that nodes move in a same direction behind the tour guide with a constant speed of $2 \mathrm{~m} / \mathrm{s}$ which is the average walking speed.

During this trip, the main factor that can affect the communication reliability is number of nodes and number of packets being sent by tour guide. So, we take different number of nodes i.e. 5, 10, 15, 20, 25 and 30. And also, different numbers of packets to be send i.e. 50, 100, 150, 200, $250,300,350,400,450$ and 500 . The direct sequence spread spectrum (DSSS) rate is $11 \mathrm{Mbps}$. As the nodes move in a same direction so Gauss-Markov Mobility Model is used as a mobility model. Data rate is taken as $2200 \mathrm{kbps}$, packet size is taken as 4096 bytes and interval between the packets transmission is taken as $0-0.015$. Simulation area is taken as $1000 * 1000 \mathrm{~m}$ and simulation time is 180 seconds. Network Simulator NS-3 is used for simulations [14]. 


\section{SIMULATION RESULTS}

Simulation results are presented on the three sections that follow. In each section behavior of each protocol is compared based on different number of nodes and packets at $11 \mathrm{Mbps}$ Direct sequence spread spectrum (DSSS) Rate. Performance metrics for the evaluation of the three routing protocols (DSDV, AODV and OLSR) are total energy consumption, throughput and packet delivery ratio (PDR).

\subsection{Packet Delivery Ratio}

It is the ratio between the numbers of packets originated by the application layer and the number of packets received by the sinks at the destination. It describes the loss rate which in turn affects the maximum throughput that the network can support. It represents the reliability of the communication.

In Figure 2 AODV and OLSR outperforms DSDV in every case. OLSR shows best performance when no. of packets is 500 resulting 81.65\% PDR. At 100, 150, 200 and 400 packets AODV outperforms OLSR, but in all other cases OLSR shows best results.

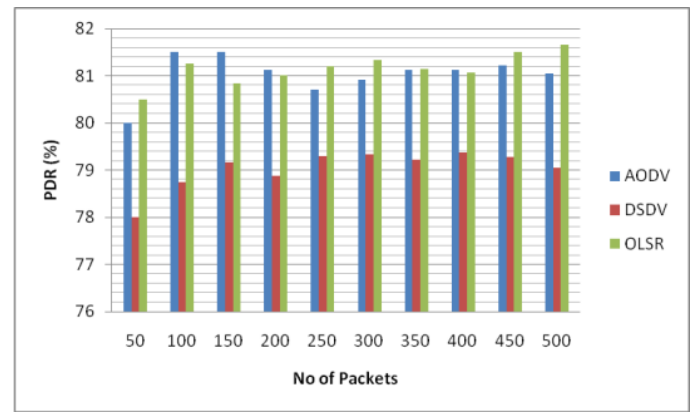

Fig 2: PDR vs. No. of Packets sends when nodes are 5

Figure 3 shows that when no. of nodes is increased to 10 AODV gives worst results. When number of packets is 150 , 200, 250, 500, DSDV outperforms OLSR, but at high traffic OLSR performs best from two of them.

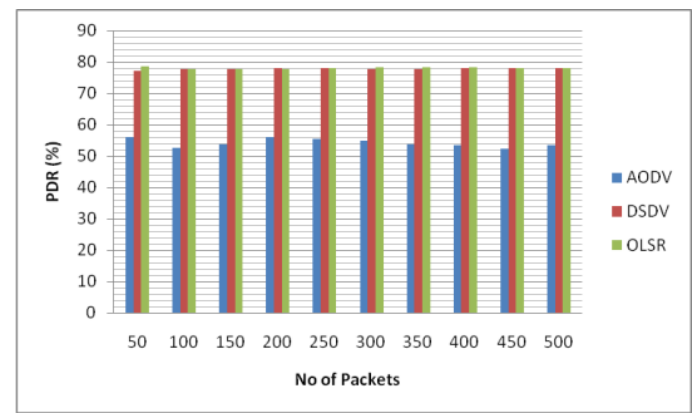

Fig 3: PDR vs. No. of Packets sends when nodes are 10

In Figure 4 AODV shows worst performance. OLSR outperforms DSDV in every case except when number of packets is 50 and 100 .

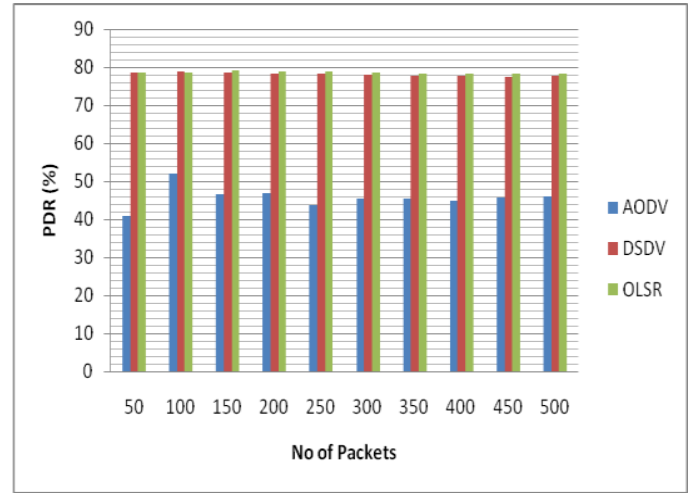

Fig 4: PDR vs. No. of Packets sends when nodes are 15

Figure 5 show that DSDV and OLSR outperform AODV in every case. OLSR outperforms DSDV only when number of packets are 50 .

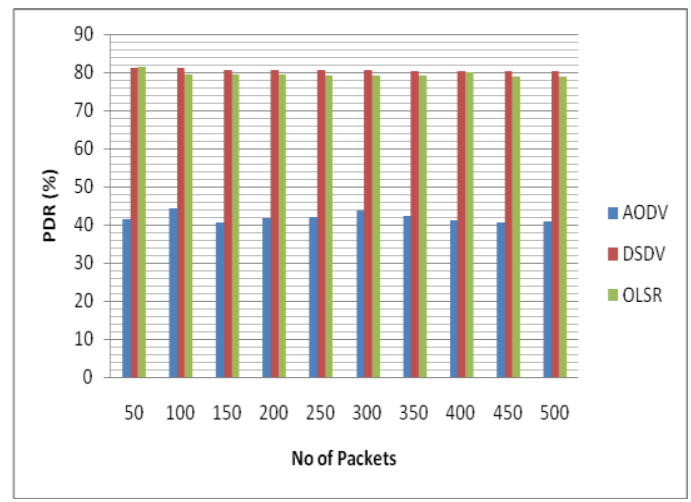

Fig 5: PDR vs. No. of Packets sends when nodes are 20

In Figure 6 it is shown that in every case OLSR outperforms DSDV and AODV.

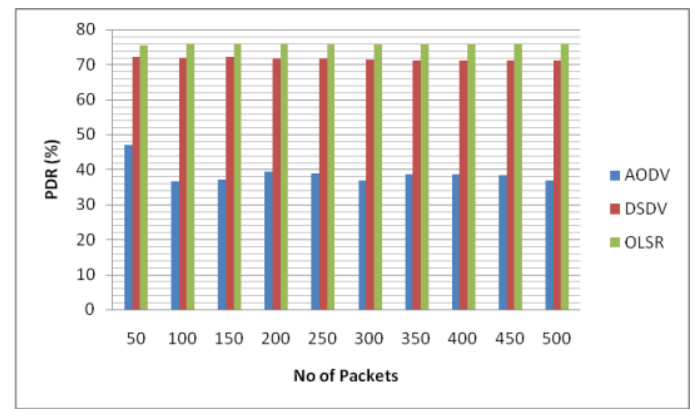

Fig 6: PDR vs. No. of Packets sends when nodes are 25

In Figure 7 OLSR and DSDV outperforms AODV in every case and OLSR performance is better than DSDV. 


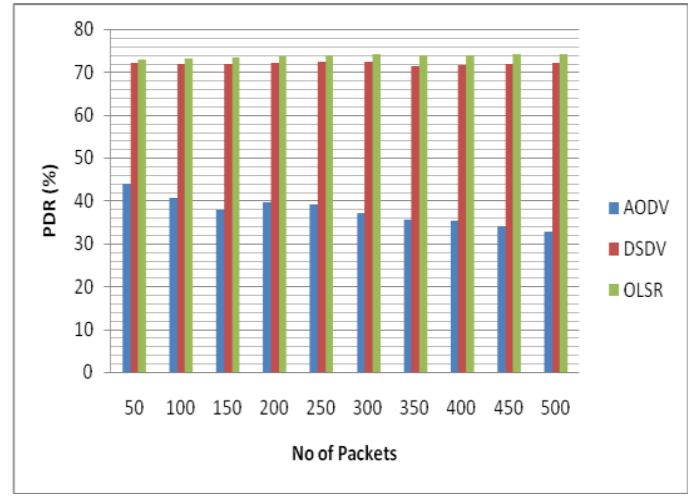

Fig 7: PDR vs. No. of Packets sends when nodes are 30

\subsection{Energy consumption}

Energy consumption is very important factor as in our scenario nodes may not have the chance to recharge their portable devices. In following figures, energy consumption is compared between these protocols at 11 Mbps DSSS Rates.

In Figure 8 it is shown that AODV consumes maximum energy and DSDV consumes minimum.

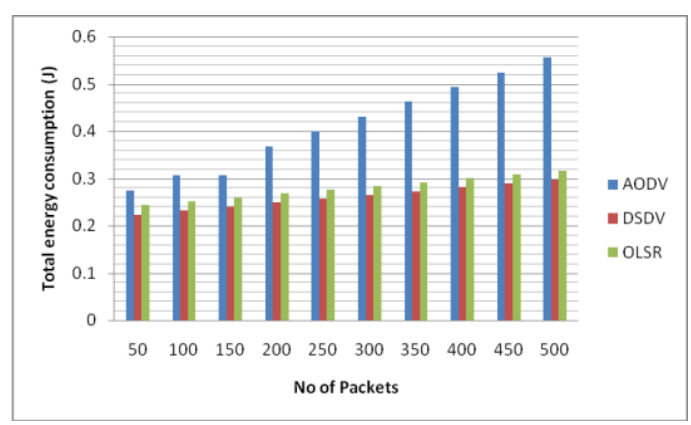

Fig 8: Total energy consumed vs. No. of Packets sends when nodes are 5

Figure 9 shows that AODV consumes maximum energy and DSDV consumes minimum.

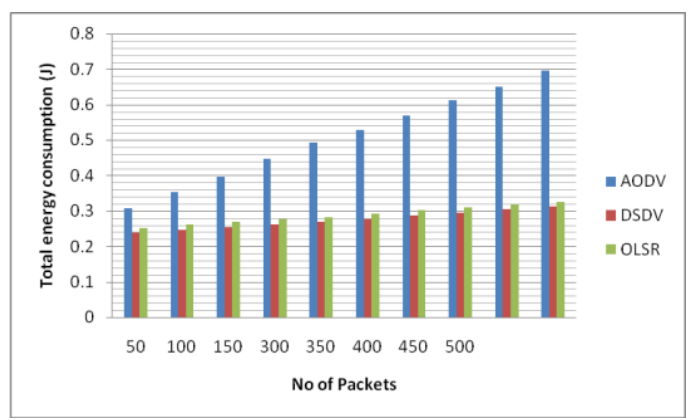

Fig 9: Total energy consumed vs. No. of Packets sends when nodes are 10

In Figure 10 it is shown that AODV consumes maximum and DSDV consumes minimum energy.

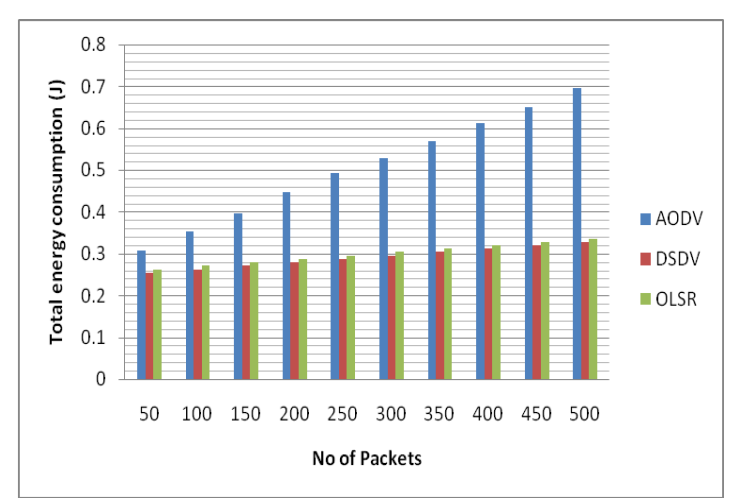

Fig 10: Total energy consumed vs. No. of Packets sends when nodes are 15

In Figure 11 AODV consumes maximum energy and OLSR consumes minimum energy.

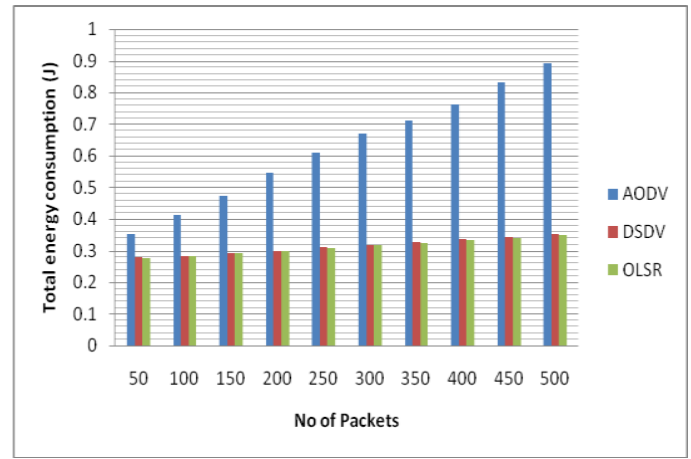

Fig 11: Total energy consumed vs. No. of Packets sends when nodes are 20

Figure 12 shows that AODV consumes maximum energy and OLSR consumes minimum energy.

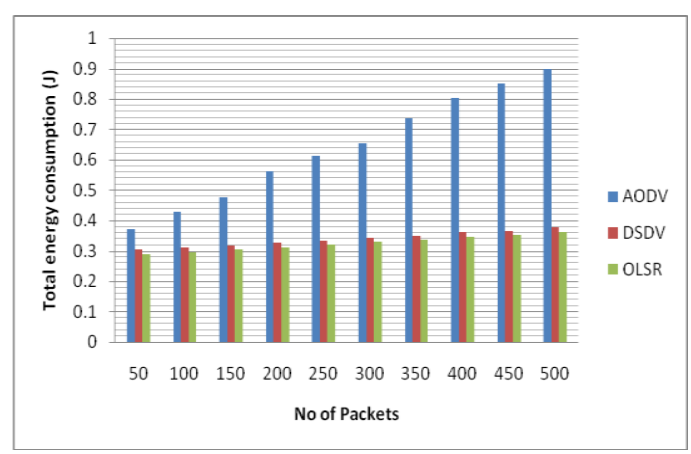

Fig 12: Total energy consumed vs. No. of Packets sends when nodes are 25

Figure 13 shows that AODV consumes maximum energy and OLSR consumes minimum. 


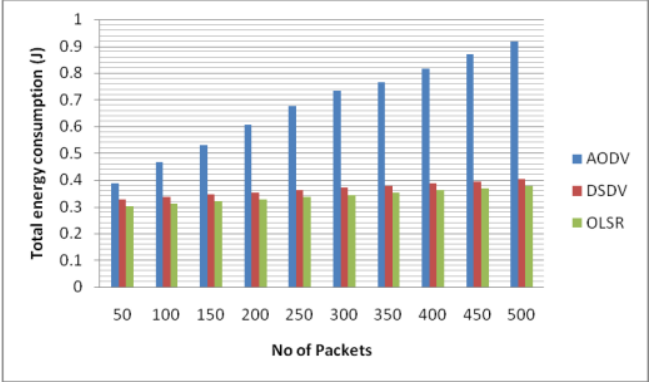

Fig 13: Total energy consumed vs. No. of Packets sends when nodes are 30

\subsection{Throughput}

It is the average rate of successful message delivery over a communication channel. In following figures, average delay is compared between these protocols at $11 \mathrm{Mbps}$ DSSS Rates.

Figure 14 shows that when number of packets is 150 , DSDV results more throughput than AODV and OLSR. Otherwise DSDV shows worst performance in every case. AODV outperforms OLSR at 100, 250, 300, 400, and 450 and in all other cases OLSR outperforms AODV.

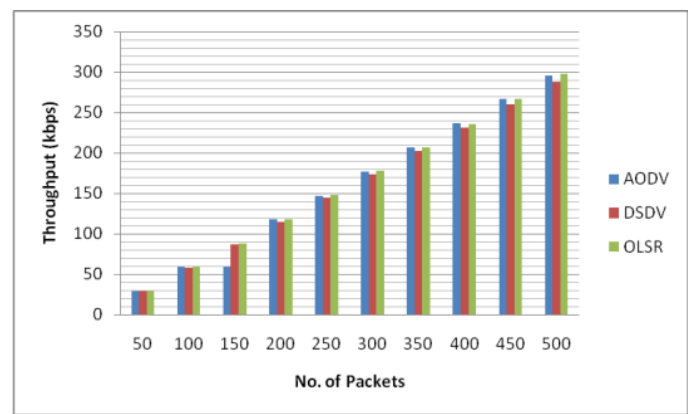

Fig 14: Throughput vs. No. of Packets sends when nodes are 5

Figure 15 shows that when number of packets is 150,200 , 250 and 500 DSDV outperforms OLSR and in all other cases OLSR shows best results.

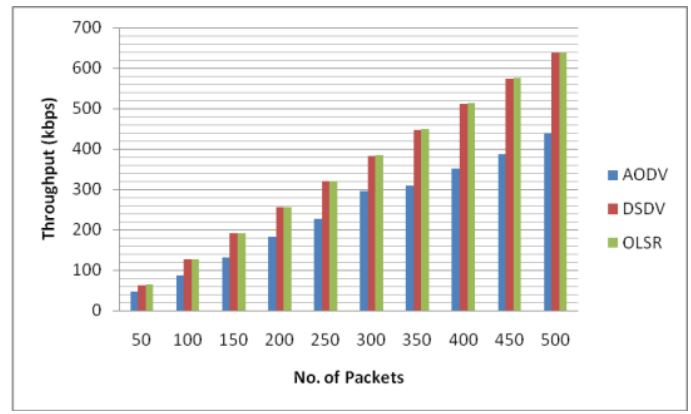

Fig 15: Throughput vs. No. of Packets sends when nodes are 10

Figure 16 show that when number of packets is 50 and 100 DSDV outperforms OLSR.

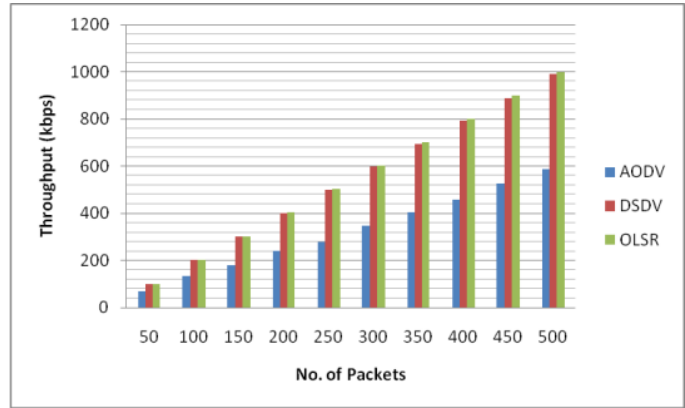

Fig 16: Throughput vs. No. of Packets sends when nodes are 15

In Figure 17 AODV shows worst performance. DSDV outperforms OLSR in every case.

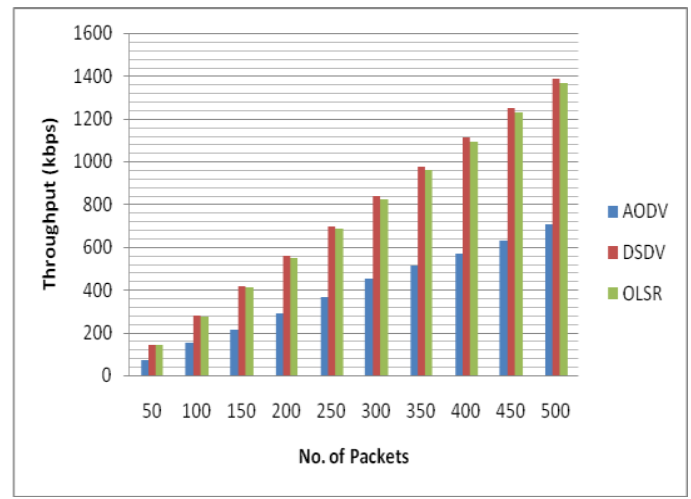

Fig 17: Throughput vs. No. of Packets sends when nodes are 20

In Figure 18 OLSR outperforms DSDV in every case and AODV shows worst performance in all cases.

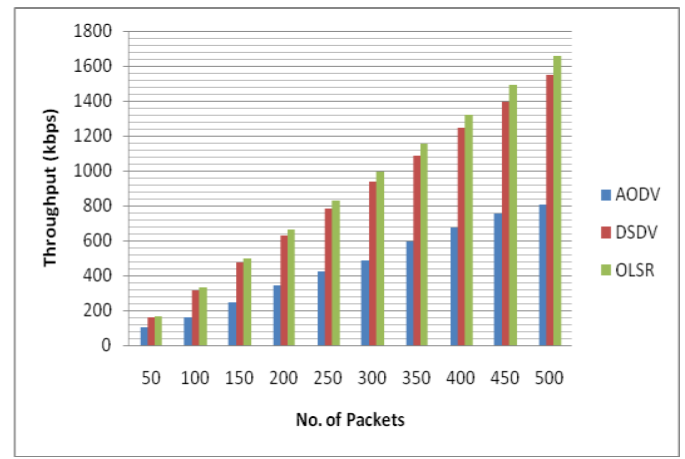

Fig 18: Throughput vs. No. of Packets sends when nodes are 25

Figure 19 shows that OLSR outperforms DSDV in every case and AODV shows worst results in all cases. 


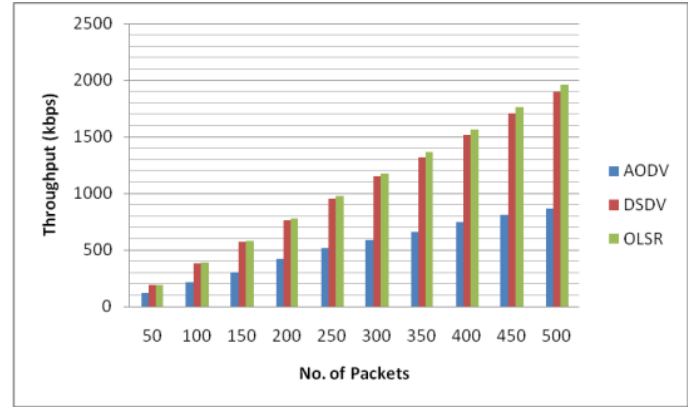

Fig 19: Throughput vs. No. of Packets sends when nodes are 30

\section{CONCLUSION}

During the case of PDR, AODV has shown the $81.5 \%$ best PDR performance only when, number of nodes is 5 and number of packets is 100 and 150 , but in all other cases, the other two protocols always outperform AODV. OLSR has best performance among them with $81.65 \%$ best PDR when number of nodes are 5 and number of packets are 500 where as DSDV shows $81.2632 \%$ best PDR when number of nodes are 20 and number of packets are 100 . So according to this scenario, it is concluded that when traffic is slow then AODV shows best results, when traffic is medium then DSDV shows best results and at high traffic OLSR shows best results.

During the case of total energy consumption, AODV never shows best results and in all other cases DSDV and OLSR outperforms AODV. DSDV and OLSR have quite same performance but OLSR with some better results than DSDV at high traffic.

Further, in case of throughput, AODV shows best results when number of nodes is 5 and number of packets is 200 and 400. OLSR and DSDV have quite same performance but OLSR has $1959.34 \mathrm{kbps}$ the highest throughput where as DSDV has $1900.72 \mathrm{kbps}$ the highest throughput when number of nodes is 30 and number of packets is 500 and these protocols always outperform AODV.

\section{REFERENCES}

[1] Khaleel Khan, Ur Rahman Khan, Rafi u Zaman, A. Venugopal Reddy," Performance Comparison of OnDemand and Table Driven Ad Hoc Routing protocols Using NCTUns", UKSIM: IEEE Tenth International Conference on Computer Modeling and Simulation, vol.1, Issue - 3, pp. 336 - 341, April 2008.

[2] M. Amnai, Y. Fakhri and J. Abouchabaka, "Qos Routing and Performance Evaluation for Mobile Ad Hoc Networks using OLSR Protocol," Inter. Journal of Ad hoc, Sensor and Ubiquitous Computing (IJASUC), vol. 2, no. 2, June 2011.

[3] Yossef Saadi, Said El Kafhali, Abdelkrim Haqiq and Bouchaib Nassereddine, "Simulation Analysis of Routing Protocols using Manhattan Grid Mobility Model in MANET," International Journal of Computer Applications, vol. 45, no. 23, May 2012.
[4] Hong Jiang, J. J. Garcia-Luna-Aceves, "Performance comparison of three routing protocols for ad hoc networks," Int. Conf. on Computer Communications and Networks, pp. 547-554, June 2001.

[5] E. Ahvar and M. Faithy, "Performance Evaluation of Routing Protocols For High Density Ad Hoc Networks based on Qos by GlomoSim Simulator," In Proceedings of World academy of science and technology, vol. 23, no. 1 , Aug. 2007

[6] Mehdi Barati, Kayvan Atefi, Farshad Khosravi and Yashar Azab Dafia, "Performance Evaluation of Energy Consumption for AODV and DSR Routing Protocols in MANET," Inter. Conf. on Computer and Information Science (ICCIS), pp. 636-642, 2012.

[7] Sunil Kr. Maakar, Sudesh Kumar and Amit Nain, "Performance analysis of Proactive and Reactive Routing Protocols using TCP Traffic for Freeway and Random Waypoint Mobility Model," International Journal of Computer Applications, vol. 43, no. 7, pp. 1318, April 2012.

[8] C. Mbarushimana, A. Shahrabi, "Comparative Study of Reactive and Proactive Routing Protocols Performance in Mobile Ad Hoc Networks," Advanced Information Networking and Applications Workshops (AINAW), pp. 679-684, 2007.

[9] C. E. Perkins and E. M. Royer, "Ad Hoc On-Demand Distance Vector Routing," In Proceedings of IEEE Workshop on Mobile Computing Systems and Applications, pp. 90-100, February 1999.

[10] S.R. Das , R. Castaneda, J. Yan R. Sengupta, "Comparative performance evaluation of routing protocols for mobile adhoc networks," $7^{\text {th }}$ Int. Conf. on Computer Communications and Networks (IC3N), pp.153-161, Oct. 1998.

[11] M. S. Coroson and J. Macker, "Mobile ad hoc networking (MANET): routing protocol performance issues and evaluation considerations," IETF MANET, RFC 2501, 1999.

[12] I. Chlamtac, M. Conti, J.J.-N. Liu, "Mobile ad hoc networking: imperatives and challenges," $\mathrm{Ad} H \mathrm{Hoc}$ Networks, vol. 1, no. 1, pp. 13-64, 2003.

[13] C. Siva Ram Murthy and B.S. Manoj, Ad Hoc Wireless Networks. India: PEARSON Education, 2004.

[14] ns-3. [Online]. Available: http://www.nsnam.org/

[15] C. E. Perkins and P. Bhagwat, "Highly Dynamic Destination-Sequenced Distance-Vector Routing (DSDV) for Mobile Computers," In Proceedings of ACM SIGCOMM, pp. 234-244, August 1994.

[16] T. H. Clausen, G. Hansen, L. Christensen \& G Behrmann, "The Optimized Link State Routing Protocol, Evaluation Through Experiments and Simulation," In Proceedings of IEEE Symposium on Wireless Personal Mobile Communications, pp. 841-846, September 2001. 